INPLASY

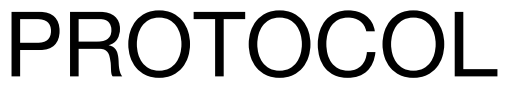

To cite: Clemente et al. Variability of internal and external load and technical/ tactical outcomes during small-sided soccer games: A systematic review. Inplasy protocol 202130080. doi: 10.37766/inplasy2021.3.0080

Received: 22 March 2021

Published: 22 March 2021

Corresponding author:

Filipe Manuel Clemente

filipe.clemente5@gmail.com

Author Affiliation:

Escola Superior Desporto e Lazer, Instituto Politécnico de Viana do Castelo, Rua Escola Industrial e Comercial de Nun'Álvares, 490

Support: None.

Review Stage at time of this submission: Data analysis.

Conflicts of interest:

None declared.

\section{Variability of internal and external load and technical/tactical outcomes during small-sided soccer games: A systematic review}

Clemente, FM1; Aquino, R²; Praça, G3; Rico-González, M4; Oliveira, R5; Silva, AF6; Sarmento, $\mathrm{H}^{7}$; Afonso, J8.

Review question / Objective: This systematic review aimed to: (1) identify the studies that have examined the intra- and intersession bouts'variability levels regarding the internal and external load and technical/tactical outcomes during SSGs; and (2) summarize the main evidence.

Condition being studied: Players exposed to a minimum of two bouts/sets of a SSG (within- or between-sessions). Thus, the same game was made at least twice in a single session or at least one time in two different training sessions.

Information sources: Electronic databases (PubMed, SPORTDiscus, Cochrane and Web of Science - core collection) were searched for relevant publications prior to the February 9 of 2021.

INPLASY registration number: This protocol was registered with the International Platform of Registered Systematic Review and Meta-Analysis Protocols (INPLASY) on 22 March 2021 and was last updated on 22 March 2021 (registration number INPLASY202130080).

\section{INTRODUCTION}

Review question / Objective: This systematic review aimed to: (1) identify the studies that have examined the intra- and inter-session bouts'variability levels regarding the internal and external load and technical/tactical outcomes during
SSGs; and (2) summarize the main evidence.

Rationale: Small-sided games (SSGs) are widely used in the soccer training context. However, some of the typical outcomes related to human response during these games (namely internal and external load) 
may be highly variable within- and between-sessions for similar conditions of practice. Thus, the study of intra- and interbouts variability in response to SSGs is progressively growing.

Condition being studied: Players exposed to a minimum of two bouts/sets of a SSG (within- or between-sessions). Thus, the same game was made at least twice in a single session or at least one time in two different training sessions.

\section{METHODS}

Search strategy: Electronic databases (PubMed, SPORTDiscus, Cochrane and Web of Science - core collection) were searched for relevant publications prior to the February 9 of 2021. Keywords and synonyms were entered in various combinations: title (i.e., "Soccer" OR "Football") AND title ("small-sided" OR "SSG" OR "conditioned") AND in the title, abstract or keywords ("varia*" OR "reproducibility" OR "repeatability" OR "reliability"). Additionally, the reference lists of the studies retrieved were manually searched to identify potentially eligible studies not captured by the electronic searches. Finally, an external expert has been contacted in order to verify the final list of references included in this scoping review in order to understand if there was any study that was not detected through our research. Possible errata was searched for each included study.

Participant or population: Soccer players of any age or sex, with regular training practice and without major injury or illness.

Intervention: A minimum of two bouts/sets of a SSG (within- or between-sessions). Thus, the same game was made at least twice in a single session or at least one time in two different training sessions. AND - The exact same conditions of practice (e.g., same teams, same format of play) were made between repetitions.

Comparator: The comparators are the different bouts/sets of each SSG.
Study designs to be included: Repeated measures design with the same players and teams.

Eligibility criteria: Inclusion criteria: (i) Soccer players of any age or sex, with regular training practice and without major injury or illness; (ii) A minimum of two bouts/sets of a SSG (within- or betweensessions). Thus, the same game was made at least twice in a single session or at least one time in two different training sessions AND The exact same conditions of practice (e.g., same teams, same format of play) were made between repetitions; (iii) The comparators are the different bouts/sets of each SSG; (iv) Any measure of variability (e.g., ICC, CV, etc.) or any metrics that, combined, afford calculation of variability (e.g., mean $\pm S D$, mean $\pm S E M)$. One of the following outcomes should be included: Internal load [heart rate; blood lactate concentrations; rate of perceived exertion]; External load [total distance; distances between 19.8 and $25 \mathrm{~km} / \mathrm{h}$; distances > 25 $\mathrm{km} / \mathrm{h}$; accelerations and decelerations]; Technical actions [passes; receptions; ball touches; shots]; Tactical behavior [attacking behaviors; defensive behaviors]; (v) Repeated measures design with the same players and teams; (vi) Original research published in peer-review journals, restricted to English, Portuguese and Spanish and no limited to date. Exclusion criteria: (i) Sports other than soccer (e.g., rugby, American football, handball, volleyball, futsal, basketball). Players with major injuries or illness; (ii) SSGs with single bout/set; The bouts/sets changes the constraints (e.g., play format, court dimensions) or conditions (change teams and players within or between-sessions); The conditions changes by any exercise or test (e.g., inducing mental or physical fatigue) made between repetitions occurring in the same session; (iii) Single bout/set of an SSG; (iv) Does not present a measure of variability (e.g., ICC, CV, etc.) or any metrics that, combined, afford calculation of variability (e.g., mean $\pm S D$, mean \pm SEM); Does not present at least one of the following outcomes; Examples of exclusion: Well-being parameters related to measures of fatigue, stress, mood, 
recovery, sleep quality or others; Psychological or sociological outcomes as enjoyment or cohesion; Readiness parameters as heart rate variability, neuromuscular capacity or others; (v) No repeated measures design with the same players and teams; Experimental studies analyzing the effects of SSGs training protocols on fitness/technical or tactical variables; (vi) Written in other language than English, Portuguese or Spanish. Other article types than original (e.g., reviews, letters to editors, trial registrations, proposals for protocols, editorials, book chapters and conference abstracts).

Information sources: Electronic databases (PubMed, SPORTDiscus, Cochrane and Web of Science - core collection) were searched for relevant publications prior to the February 9 of 2021.

Main outcome(s): The intraclass correlation coefficient (ICC) and/or typical error of measurement (TEM) (\%) and/or coefficient of variation (CV) (\%) and/or standard error of measurement (SEM) values were extracted from the original articles regarding the following measures: (i) internal load [e.g., heart rate; blood lactate concentrations; rate of perceived exertion]; (ii) external load [e.g., total distance; distances between 19.8 and $25 \mathrm{~km} / \mathrm{h}$; distances > $25 \mathrm{~km} / \mathrm{h}$; accelerations and decelerations]; (iii) technical actions [e.g., passes; receptions; ball touches; shots]; and (iv) tactical behavior [e.g., attacking behaviors; defensive behaviors].

Data management: A data extraction was prepared in Microsoft Excel sheet (Microsoft Corporation, Readmon, WA, USA) in accordance with the Cochrane Consumers and Communication Review Group's data extraction template (Group, 2016). The Excel sheet was used to assess inclusion requirements and subsequently tested for all selected studies. The process was independently conducted by two of the authors (FMC and HS). Any disagreement regarding study eligibility was resolved by a third author (JA). Full text articles excluded, with reasons, were recorded. All the records were stored in the sheet.
Quality assessment / Risk of bias analysis: Adapted version of STROBE assessment was used to evaluate the included articles' eligibility (O'Reilly et al., 2018). Any disagreement was discussed and solved by consensus decision. Each of ten items was qualified using numerical codification ( $1=$ considered or 2 = non-considered). Those studies with more than 7 complete items (score of 7 is not included), are considered as a low risk of bias.

Strategy of data synthesis: The intraclass correlation coefficient (ICC) and/or typical error of measurement (TEM) (\%) and/or coefficient of variation (CV) (\%) and/or standard error of measurement (SEM) values were extracted from the original articles regarding the following measures: (i) internal load [e.g., heart rate; blood lactate concentrations; rate of perceived exertion]; (ii) external load [e.g., total distance; distances between 19.8 and 25 $\mathrm{km} / \mathrm{h}$; distances > $25 \mathrm{~km} / \mathrm{h}$; accelerations and decelerations]; (iii) technical actions [e.g., passes; receptions; ball touches; shots]; and (iv) tactical behavior [e.g., attacking behaviors; defensive behaviors]. Additionally, the following information was extracted from the included studies: (i) number of participants (n), age (years), competitive level (if available) and sex; (ii) the SSGs format, pitch configuration and other information about tactical conditions; (iii) number of repetitions and sessions analyzed; (iv) training regimen (work duration, work intensity, modality, relief duration, relief intensity, repetitions and series, between-set recovery); and (v) instruments used to monitor the load and the errors reported to those instruments.

Subgroup analysis: None.

Sensitivity analysis: None.

Language: English.

Country(ies) involved: Portugal; Brazil; Spain.

Keywords: football; performance; conditioned games; physiological responses; motor skills. 
Contributions of each author:

Author 1 - Filipe Manuel Clemente - The author lead the project, run the data search and methodological assessment, and wrote and revised the original manuscript.

Author 2 - Rodrigo Aquino - The author wrote and revised the original manuscript.

Author 3 - Gibson Praça - The author wrote and revised the original manuscript.

Author 4 - Markel Rico-González - The author run the data search and methodological assessment and wrote and revised the original manuscript.

Author 5 - Rafael Oliveira - The author wrote and revised the original manuscript. Author 6 - Ana Filipa Silva - The author Wrote and revised the manuscript.

Author 7 - Hugo Sarmento - The author wrote and revised the original manuscript. Author 8 - José Afonso - The author run the data search and methodological assessment and wrote and revised the original manuscript. 